\title{
MONET Special Issue on Towards Future Ad Hoc Networks: Technologies and Applications (I)
}

\author{
Jun Zheng ${ }^{1} \cdot$ Wei Xiang ${ }^{2} \cdot$ Pascal Lorenz ${ }^{3} \cdot$ Shiwen Mao ${ }^{4}$ \\ Published online: 9 January 2020 \\ (C) Springer Science+Business Media, LLC, part of Springer Nature 2020
}

An ad hoc network is a wireless system in which network nodes are connected via wireless links and cooperate to self-organize into a network with no need for any infrastructure such as access points or base stations. Ad hoc networks cover a variety of paradigms such as mobile ad hoc networks (MANETs), wireless sensor networks (WSNs), vehicular ad hoc networks (VANETs), underwater networks, airborne networks, underground networks, personal area networks, and home networks. These types of networks promise a wide range of applications in civilian, commercial, and military areas. However, the distributed and multi-hop nature of ad hoc networking as well as the highly dynamic topology of ad hoc networks due to node mobility have presented many formidable technical challenges, such as scalability, quality of service, reliability and security, and energy efficiency, which have stimulated a considerable amount of research activities in this broad area in recent years.

This special issue includes six papers that report recent research advances in the area of ad hoc networks, among which four papers are selected from AdHocNets 2018 and two are selected from open call. In the first paper, "Prediction Based Vehicular Caching: Where and What to

\author{
Jun Zheng \\ junzheng@seu.edu.cn \\ Wei Xiang \\ wei.xiang@jcu.edu.au \\ Pascal Lorenz \\ lorenz@ieee.org \\ Shiwen Mao \\ smao@auburn.edu \\ Southeast University, Nanjing, China \\ James Cook University, Townsville, Australia \\ University of Haute-Alsace, Mulhouse, France \\ 4 Auburn University, Auburn, Alabama, USA
}

Cache", Zhang et al. proposed a prediction based vehicular caching scheme by exploiting the performance of a vehicular caching scheme where the role of moving vehicles changes from service consumers in traditional networks to service providers and service consumers. Specifically, an optimization problem is firstly formulated by exploring the relationship of caching vehicles and mobile users to optimize the network energy efficiency. The nonconvex optimization problem is solved by decomposing it into a nonlinear programming problem. By applying the Lyapunov method and autoregressive neural network (ANN), an online caching decision algorithm is further proposed to make caching decisions. Simulations Results show that the vehicular caching scheme can obviously improve network energy efficiency with different requests. The comparison between online and offline caching also shows the necessity of online caching decision making due to its benefit in resource utilization where the system gain increases from $8.4 \%$ to $59.24 \%$.

In the second paper, "Task Allocation in Semi-Opportunistic Mobile Crowdsensing: Paradigm and Algorithms," Gong et al. proposed a new mobile crowdsensing paradigm, named semiopportunistic sensing, aiming to achieve both high task coverage and low worker employment fee. In this paradigm, each worker can provide multiple candidate moving paths for his/her trip, among which the service platform chooses one for the worker to undertake task(s). The platform selects workers and assigns tasks to them with an objective to optimize total task quality under the platform's incentive budget and workers' task performing time constraints. The authors formulated the task allocation problem, proved its NP-hardness, and presented two efficient heuristic algorithms. The first heuristic, named Best Path/Task first algorithm (BPT), always chooses the best path and task in a greedy manner. The second heuristic, named LP-Relaxation based algorithm (LPR), assigns paths and tasks with the largest values according to the LP-relaxation. Experiment results show that the proposed semi-opportunistic sensing paradigm can significantly improve total task quality compared with opportunistic sensing. 
In the third paper, "Connectivity Based $k$-Coverage Hole Detection in Wireless Sensor Networks," Yan et al. proposed a connectivity based k-coverage hole detection algorithm and adopted Rips complex in homology theory to model a wireless sensor network (WSN). A simplicial complex reduction algorithm is first proposed to simplify the network topology by vertex and edge deletion, while keeping the homology intact. Then a connectivity-based algorithm is proposed for discovering the boundary cycles of non-triangular k-coverage holes. The proposed algorithm consists of two stages, one is for 1-coverage hole-detection and the other is for coverage degree reduction. In the 1-coverage hole-detection stage, boundary cycles of 1-coverage holes are found. In the coverage degree reduction stage, an independent covering subset of nodes in the covered region is found and the nodes are set to a dormant state to decrease the coverage degree of the target region by one. Simulation results show that more than $95 \%$ of non-triangular k-coverage holes can be accurately detected by the proposed hole-detection algorithm.

WSNs are extensively used in monitoring applications such as humidity and temperature sensing in smart buildings. However, limited energy in a sensor's battery prevents a sensor from operating in a long term. Energy harvesting has been considered as an effective approach to address the energy limitation in sensor devices and adaptive power management is essential to energy harvesting in WSNs. The fourth paper, “Autonomous Energy Management System Achieving Piezoelectric Energy Harvesting in Wireless Sensors," by Kassan et al., presents a self-management of energy based on a Proportional Integral Derivative (PID) controller, which tunes the Microprocessor Controller Unit (MCU) to adaptively control the sensor modes based on the existing vibration in the environment in order to extend the lifetime of sensor nodes.

In the fifth paper, "Design of a Practical WSN based Fingerprint Localization System," Zou et al. proposed a practical WSN based fingerprint localization system. The proposed system covers both indoor and outdoor scenarios, and can meet the demand for seamless localization. Four measures are introduced in the system to improve fault tolerance and system efficiency, including a traffic regulation based radiomap (TRRM) establishing method, a full-overlapping clustering strategy, an adaptive feature space (AFS) algorithm, and a praxeological tracking algorithm. Hardware experiments using smart phones show that the proposed localization system can achieve a positioning accuracy of within $5 \mathrm{~m}$ in pedestrian tests and within $10 \mathrm{~m}$ in driving tests.

In the last paper, "Indoor Localization Using Smartphone Magnetic and Light Sensors: A Deep LSTM Approach," Wang et al. presented DeepML, a deep long short-term memory (LSTM) based system for indoor localization using magnetic and light sensors on smart-phones. The authors experimentally verified the feasibility of using bimodal data from magnetic and light sensors for indoor localization for closed environments where there is no ambient light. Then they designed the DeepML system, which first builds bimodal images by data preprocessing, and then trains a deep LSTM network in the offline phase. Newly received magnetic field and light data are then exploited for estimating the location of the mobile device using a probabilistic method. The effectiveness of the proposed DeepML system is verified by the extensive experiments.

We thank all the authors for their contributions to this special issue. We are grateful to all reviewers for their time and efforts in reviewing all the papers and providing valuable review comments. We also thank Eliška Vlčková, EAI Managing Editor, Rolissa Atienza and Jed Joseph Adel, Springer JEO Assistant, Leonora Mariño Panday, Springer Production Coordinator, and all other production staff for their support during the publication process.

It is our hope that the papers included in this special issue present recent research advances in ad hoc networks, and will become an important reference for researchers and practitioners in the area. Finally, we hope that the readers will find this special issue timely and informative.

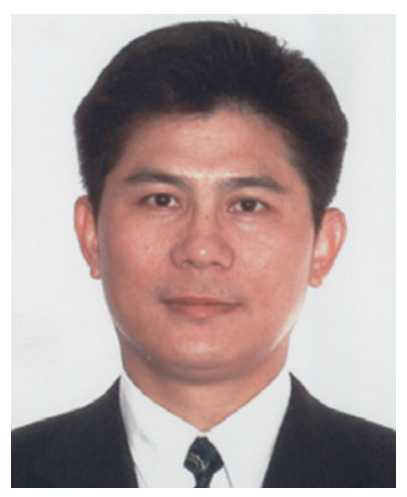

JunZheng(junzheng@ seu.edu.cn) received the $\mathrm{Ph}$. D. degree in electrical and electronic engineering from The University of Hong Kong, Hong Kong, in 2000. $\mathrm{He}$ is a Full Professor at the National Mobile Communications Research Laboratory, Southeast University (SEU), Nanjing, Jiangsu, China. Before joining SEU, he was with the School of Information Technology and Engineering, University of Ottawa, Ottawa, ON, Canada. He has coauthored (first author) two books published by Wiley-IEEE Press, and has co-authored nearly 200 technical papers in refereed journals and peer-reviewed conference proceedings. He is the co-recipient of an ICC 2014 Best Paper Award, a WCSP 2018 Best Paper Award, and a WiCON 2017 Best Student Paper Award. His current research interests include vehicular ad hoc networks, mobile communication networks, wireless sensor networks, focused on network architectures and protocols. Dr. Zheng serves as a Technical Editor of IEEE Communications Magazine and is an editorial board member of several refereed journals. He has co-edited a number of special issues for different refereed journals and magazines, including IEEE Communications Magazine, IEEE Network, and IEEE Journal on Selected Areas in Communications, all as Lead Guest Editor. He has served as the founding General Chair of AdHocNets'09, General Chair of AccessNets'07/AdHocNets'18/19, and TPC or Symposium Co-Chair of a number of international conferences and symposia, including IEEE ICC 2009/2011/2015/ 2021 and GLOBECOM 2008/2010/2012/2018/2019. He has also served as a technical program committee member for a number of international conferences and symposia. He is a senior member of the IEEE, IEEE Communications Society, and IEEE Vehicular Technology Society. He also serves as Chair of IEEE Vehicular Technology Society Nanjing Chapter, 
and Chair of IEEE ComSoc Communications Switching and Routing Technical Committee.

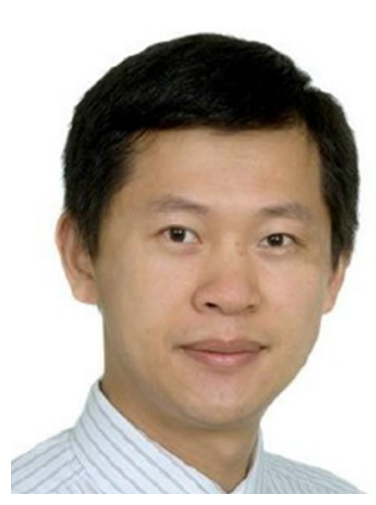

Wei Xiang (wei.xiang @jcu.edu.au) is the Foundation Chair and Head of Discipline of Internet of Things Engineering at James Cook University, Cairns, Australia. Due to his instrumental leadership in establishing Australia's first accredited Internet of Things Engineering degree program, he was selected into Pearcy Foundation's Hall of Fame in October 2018. He is an elected Fellow of the IET in UK and Engineers Australia. He received the TNQ Innovation Award in 2016, and Pearcey Entrepreneurship Award in 2017, and Engineers Australia Cairns Engineer of the Year in 2017. He was a co-recipient of four Best Paper Awards at WiSATS'2019, WCSP'2015, IEEE WCNC'2011, and ICWMC'2009. He has been awarded several prestigious fellowship titles. He was named a Queensland International Fellow (2010 2011) by the Queensland Government of Australia, an Endeavour Research Fellow (2012-2013) by the Commonwealth Government of Australia, a Smart Futures Fellow (2012-2015) by the Queensland Government of Australia, and a JSPS Invitational Fellow jointly by the Australian Academy of Science and Japanese Society for Promotion of Science (2014-2015). He is the Vice Chair of the IEEE Northern Australia Section. He was an Editor for IEEE Communications Letters (20152017), and is an Associate Editor for Springer's Telecommunications Systems. He has published over 250 peer-reviewed papers including 3 academic books and 160 journal articles. He has severed in a large number of international conferences in the capacity of General Co-Chair, TPC CoChair, Symposium Chair, etc. His research interest falls under the broad areas of communications and information theory, particularly the Internet of Things, and coding and signal processing for multimedia communications systems.

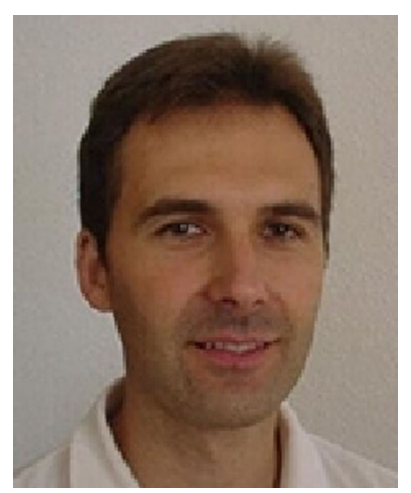

Pascal Lorenz (lorenz@ieee.org) received his M.Sc. (1990) and Ph.D. (1994) from the University of Nancy, France. Between 1990 and 1995 he was a research engineer at WorldFIP Europe and at Alcatel-Alsthom. He is a professor at the University of HauteAlsace, France, since 1995. His research interests include QoS, wireless networks and highspeed networks. He is the author/ co-author of 3 books, 3 patents and 200 international publications in refereed journals and conferences. He was Technical Editor of the IEEE Communications Magazine Editorial Board (2000-2006), IEEE Networks Magazine since 2015, IEEE Transactions on Vehicular Technology since 2017, Chair of IEEE ComSoc France (2014-2018), Financial chair of IEEE France (20172019), Chair of Vertical Issues in Communication Systems Technical Committee Cluster (2008-2009), Chair of the Communications Systems Integration and Modeling Technical Committee (2003-2009), Chair of the Communications Software Technical Committee (20082010) and Chair of the Technical Committee on Information Infrastructure and Networking (2016-2017). He has served as CoProgram Chair of IEEE WCNC'2012 and ICC'2004, Executive ViceChair of ICC'2017, TPC Vice Chair of Globecom'2018, Panel sessions co-chair for Globecom'16, tutorial chair of VTC'2013 Spring and WCNC'2010, track chair of PIMRC'2012 and WCNC'2014, symposium Co-Chair at Globecom 2007-2011, Globecom'2019, ICC 2008-2010, ICC'2014 and '2016. He has served as Co-Guest Editor for special issues of IEEE Communications Magazine, Networks Magazine, Wireless Communications Magazine, Telecommunications Systems and LNCS. $\mathrm{He}$ is associate Editor for International Journal of Communication Systems (IJCS-Wiley), Journal on Security and Communication Networks (SCN-Wiley) and International Journal of Business Data Communications and Networking, Journal of Network and Computer Applications (JNCA-Elsevier). He is senior member of the IEEE, IARIA fellow and member of many international program committees. He has organized many conferences, chaired several technical sessions and gave tutorials at major international conferences. He was IEEE ComSoc Distinguished Lecturer Tour during 2013-2014.

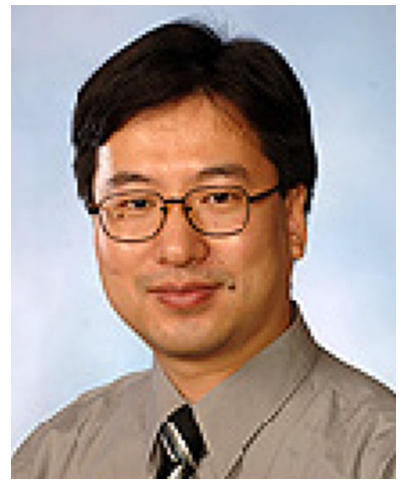

ShiwenMao(smao@ auburn.edu) received his Ph.D. in electrical and computer engineering from Polytechnic University, Brooklyn, NY in 2004. Currently, he is the Samuel Ginn Professor in the Department of Electrical and Computer Engineering, and Director of the Wireless Engineering Research and Education Center (WEREC) at Auburn University, Auburn, AL. His research interests include wireless networks, multimedia communications, and smart grid. $\mathrm{He}$ is on the Editorial Board of IEEE Transactions on Wireless Communications, IEEE Open Journal of the Communications Society, IEEE Internet of Things Journal, IEEE/CIC China Communications, IEEE Transactions on Network Science and Engineering, IEEE Transactions on Mobile Computing, IEEE Transactions on Multimedia, 
IEEE Multimedia, IEEE Networking Letters, and ACM GetMobile, among others. He received is a co-recipient of the IEEE ComSoc MMTC Best Journal Paper Award in 2019 and the Best Conference Paper Award in 2018, the Best Demo Award from IEEE SECON 2017, the Best Paper
Awards from IEEE GLOBECOM 2019, 2016, and 2015, IEEE WCNC 2015, and IEEE ICC 2013, and the 2004 IEEE Communications Society Leonard G. Abraham Prize in the Field of Communications Systems. He is a Fellow of the IEEE and a Member of the ACM. 